\title{
Optimal quantum noise cancellation with an entangled witness channel
}

\author{
Daniel W. Gould $\odot,{ }^{*}$ Min Jet Yap, Vaishali B. Adya $\odot$, Bram J. J. Slagmolen $\odot$, Robert L. Ward $\odot$, and David E. McClelland \\ OzGrav, Centre for Gravitational Astrophysics, Research School of Physics and Research School of Astronomy and Astrophysics, \\ Australian National University, Acton, Australian Capital Territory 2601, Australia
}

(Received 23 April 2021; revised 29 September 2021; accepted 4 October 2021; published 28 October 2021)

\begin{abstract}
We present the digital signal processing of a mutually entangled, two-mode squeezed state using Wiener filtering to maximize the reduction of quantum noise of a single mode. By conditioning this mode, the signal, with its directly detected entangled pair, the witness, we show quantum noise cancellation of $2 \mathrm{~dB}$ below that of the signal vacuum level. We present the frequency-dependent digital recovery of squeezed states with Wiener filtering. This filtering is particularly relevant for gravitational wave detectors which will seek to use frequencydependent squeezed states to improve their reach to the observable universe. We demonstrate the recovery of squeezed states in a configuration that replicates one which would provide optimum sensitivity improvement in a gravitational wave detector under the effects of radiation pressure noise. More generally, this technique may find application in other quantum-limited high-precision experiments such as those using optomechanical cavities.
\end{abstract}

DOI: 10.1103/PhysRevResearch.3.043079

\section{INTRODUCTION}

Wiener filtering [1] is a linear time-invariant filtering method that is used across a range of classical signal processing applications [2]. These types of filters satisfy the Wiener-Hopf equations and are calculated from observed or estimated models of correlations between a chosen signal and a witness channel in real time or in postprocessing. In other words, a Wiener filter aims to estimate the noise in a chosen signal by using an independent witness system to predict the noise in it. By subtracting this prediction from the signal, the noncorrelated residual is obtained. This residual contains a higher signal-to-noise ratio due to the reduction of the correlated noise. Wiener filtering techniques have recently seen application in both the theory and experiments involving precision measurement. Shown in [3-6], Wiener filtering has been used to obtain instantaneous estimates of position and momentum of macroscopic mechanical oscillator systems to generate conditional squeezed states in a classical-noisedominated regime. Other applications of Wiener filtering include the reduction of classical noise sources in gravitational wave detectors such as seismic noise and beam jitter noise [7-10].

In all of the above-mentioned applications, the Wiener filter has been calculated as a normalized cross-spectral density between an estimate of noise and the measured signal. In this paper, utilizing two entangled fields, we make a direct

\footnotetext{
${ }^{*}$ Corresponding author: daniel.gould@anu.edu.au

Published by the American Physical Society under the terms of the Creative Commons Attribution 4.0 International license. Further distribution of this work must maintain attribution to the author(s) and the published article's title, journal citation, and DOI.
}

measurement of the quantum noise of the witness field and use that to cancel noise on the signal field. The noise properties of the signal and witness fields, owing to their creation in a nondegenerate optical parametric oscillator, are correlated. A measurement of the witness field instantaneously results in the entangled quantity realizing itself on the signal field. Using the witness channel, we can calculate an optimal transfer function of the entangled noise, allowing the Wiener filter to account for decoherence and phase change due to dispersion. The result of this process is a filter which can successfully condition a single channel with its entangled noise in a phase-dependent manner, canceling the quantum noise of that channel.

A direct application of the measurement system described above is in quantum noise limited gravitational wave detectors [11-14]. One of the highest-impact astronomical detections of the past decade, GW170817 [15], united the newly emergent field of gravitational wave astronomy with its more mature counterparts in the electromagnetic spectrum [16]. The possibility of detecting these events or even more exotic sources increases with improved sensitivity of current and future gravitational wave detectors [17-20]. Current gravitational wave detectors are limited by quantum noise above $30 \mathrm{~Hz}[21,22]$ which is driven by the vacuum fluctuations entering through the dark port of the detector [23]. These vacuum fluctuations cause noise in two distinct ways.

At low frequencies, quantum radiation pressure noise causes a displacement uncertainty [24-27] in the position of the suspended mirrors of the interferometer [28]. At high frequencies, shot noise limits our ability to track the phase changes in the interferometer's arm length [29]. The reduction of quantum noise through the injection of squeezed light has been demonstrated and is now common place in the current generation of gravitational wave detectors [30-33]. The injection of squeezed light replaces these noisy vacuum 
fluctuations entering through the dark port with an engineered low-noise state. The production of this state is governed by Heisenberg's uncertainty principle, linking amplitude and phase. As such, the noise in both the amplitude and phase quadrature cannot be simultaneously reduced. One quadrature may have its variance reduced, but only at the expense of the other. Without further manipulation, a squeezed state is unable to reduce the quantum noise over the entire bandwidth of the detector.

Upcoming changes to gravitational wave detectors will seek to minimize quantum noise across their entire measurement band by engineering and injecting a squeezed state with a frequency-dependent rotation across its spectrum [23,3436]. The necessary frequency dependence will be produced by reflection off a low-loss optical filter cavity. This reflection causes a differential rotation of the correlated sidebands of the squeezed state, producing a state that has its squeezed quadrature rotate over its frequency band. The precise control of the length of this cavity is challenging owing to its narrow linewidth [37-39].

An alternative method of implementing frequencydependent squeezing without the additional filter cavity has been proposed in [40]. This method utilizes a two-mode Einstein-Podolsky-Rosen (EPR) entangled squeezed state to achieve the same effect as a filter cavity. In having two frequency separated but entangled fields, one field, the signal field, can resonate in the interferometer while the witness field is detuned. In detuning the witness field, its sidebands undergo rotation due to the phase response of the interferometer. This gives the squeezed state its frequency dependence and allows us to recover squeezing by conditioning the signal channel based on the noise detection of the witness field. Mathematically [40], the conditional variance of the signal state $V_{\text {cond }}$ can be described by

$$
V_{\text {cond }}=V_{s 1 s 1}+|g|^{2} V_{w 1 w 1}-g^{*} V_{s 1 w 1}-g V_{w 1 s 1},
$$

where $V_{s 1 s 1}$ and $V_{w 1 w 1}$ are the variances of the signal state and idler states, $V_{s 1 w 1}$ and $V_{w 1 s 1}$ are covariances of the signal and witness fields, and $g$ is the optimal filter or the Wiener filter, which in turn is given by

$$
g=\frac{V_{s 1 w 1}}{V_{w 1 w 1}} .
$$

Depending on the configuration of the interferometer and the purpose of the Wiener filtering, the optimal filter can be a linear combination of the signal and witness fields or it can be a frequency-dependent transfer function [22,40]. The efficacy of a linear filter and a frequency-dependent optimal filter is presented in Sec. III. Further study and demonstration of the systems and concepts described in this proposal will seek to inform future designs of continuous-wave quantum systems. We have previously demonstrated that EPR-based frequency-dependent squeezing can be achieved via a passive linear filtering of the two fields [41,42]. In this work we show that the application of digital signal processing via Wiener filter allows the optimal quantum noise reduction across the entire measurement band, a key component required for its integration in gravitational wave interferometers.

\section{EXPERIMENTAL SETUP}

The experimental verification of quantum Wiener filtering is performed with the setup described below. Further information on the control methods used in this experiment can be found in [41] together with its Supplemental Material. Figure 1 shows a simplified layout of the experiment. A pump field, produced by second harmonic generation of the main laser frequency, at a frequency of $2 \omega_{0}+\Delta$ is shown in green. The pump undergoes parametric down-conversion in an optical parametric oscillator (OPO) operating below threshold. This process generates two fields, a signal and witness pair, correlated in both the phase and amplitude quadratures: the signal field, centered about $\omega_{0}$, and the witness field centered about $\omega_{0}+\Delta$. These fields are shown in Fig. 1, inset (a), under the two resonances of the OPO. Using this method, the lower sidebands of the signal field are correlated with the upper sidebands of the witness field and vice versa. The signal and witness fields are separated by $\Delta / 2 \pi=851 \mathrm{MHz}$, which corresponds to one free spectral range of the OPO and amplified in the OPO linewidth of $12 \mathrm{MHz}$.

To demonstrate frequency-dependent squeezing and the effectiveness of the Wiener filter design, we inject the twomode squeezed state into a test cavity. In the context of the original proposal [40], this test cavity acts as the proxy for the entire interferometer. Detuning the signal and/or the witness fields from the test cavity resonance produces a frequencydependent phase difference between the entangled fields. This rotates the squeezing ellipse in a frequency-dependent manner upon the detection and digital filtering of the noise of the signal channel. The test cavity here was designed to be of length approximately equal to $0.5 \mathrm{~m}$ with a linewidth of $\gamma_{\mathrm{TC}} / 2 \pi=$ $1.35 \pm 0.07 \% \mathrm{MHz}$ to produce frequency-dependent squeezing in the megahertz regime. This cavity introduces additional loss upon resonance. The design of these parameters makes the areas of interest in this experiment in the megahertz region. The application of the design to a gravitational wave detector would require functionality in the tens of hertz region. There is no fundamental difference in this low-frequency application, our choice of higher frequency due to the convenience of building a controllable test cavity that would provide frequency-dependent squeezing in the megahertz regime in a tabletop setting.

Upon reflection off the test cavity the two modes of the EPR squeezed state are spatially separated using a mode cleaner cavity. This cavity is resonant for the signal field, transmitting it, while the witness field is promptly reflected. The length of this cavity is not actively controlled, causing differences in the transmission over time. The loss on transmission and drift of this cavity causes changes in the coherence between the witness and signal fields, an effect we account for when choosing the magnitude of the linear filter we apply it to in the subsequent power spectral density calculations.

After their separation, each of these fields is sent to a separate balanced homodyne detector as seen in Fig. 1. The local oscillator for these are generated by strong modulation, via an electro-optic modulator (EOM), of the main laser frequency. The EOM, with an applied frequency of $\Delta / 2$, is driven to maximize the desired fields of $\omega_{0}+\Delta$ and $\omega_{0}$. 


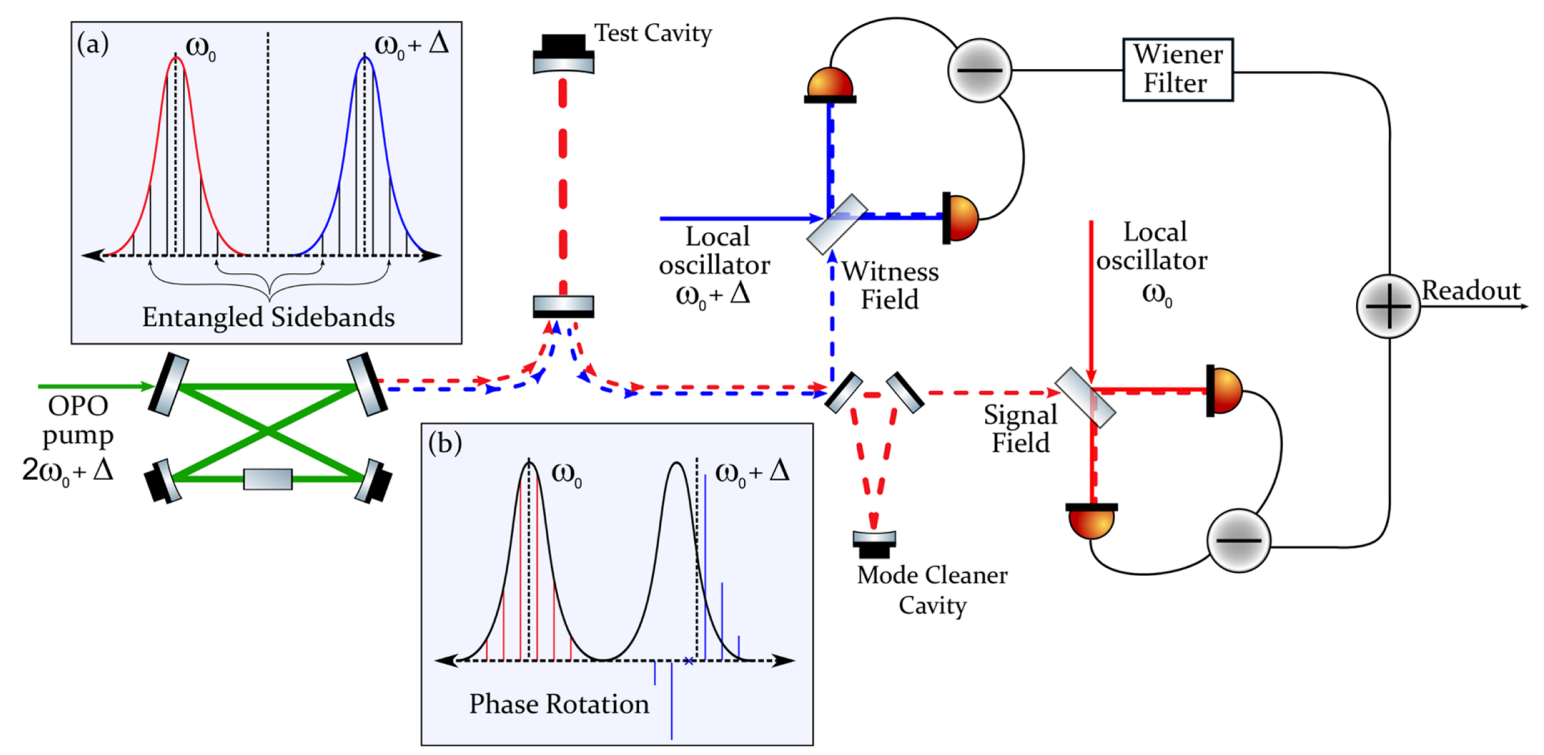

FIG. 1. Simplified schematic of the experiment. An OPO pumped with a second harmonic field at $2 \omega_{0}+\Delta$ produces the entangled sidebands of the signal (red) and witness (blue) fields within the linewidth of the separate resonances of the OPO. Inset (a) shows the frequency spacing of these fields under the OPO resonances. The entangled fields are directed to the test cavity where the signal field is resonant and the witness field is detuned. Inset (b) shows this as a frequency spacing diagram with the resonances of the test cavity shown in black. The witness field picks up a phase rotation from the phase response of the test cavity upon reflection. A Faraday isolator separates the reflected beams, sending them to a triangular mode cleaner designed such that the signal is transmitted and witness reflected, separating the two fields. The fields are detected with separate homodyne detectors for which local oscillators are generated symmetrically around $\omega+\frac{\Delta}{2}$ by strongly modulating a tap off from the main laser frequency with an EOM. A comprehensive description of the experimental design is provided in the Supplemental Material of [41].

These are filtered via the mode cleaner prior to being sent to the witness and signal homodynes. These fields are power balanced to ensure that the shot-noise levels remain constant across both signal and witness channels. When taking measurements the phase of the squeezed fields is changed relative to the local oscillators via scanning the OPO pump phase, effectively changing the phase of both signal and witness fields simultaneously and rotating the squeezed quadrature to the desired angle. Locking this angle can be achieved as seen in the Supplemental Material of [41]; however, in the results presented here the measurement time was short enough such that manual tuning of the squeezing angle was adequate.

The homodyne detectors used in this experiment were designed to have peak sensitivity in the frequency band between $10 \mathrm{kHz}$ and $10 \mathrm{MHz}$. In this region, the shot noise has a clearance from the dark noise of the detector of greater than $10 \mathrm{~dB}$ at the lower end of the spectrum, reaching $18 \mathrm{~dB}$ in the sensitivity bucket. Figure 2 shows the clearance and roll-up of the dark noise over the measurement band. Further detection losses were minimized by utilizing windowless diodes with a quantum efficiency of between $80 \%$ and $85 \%$ and ensuring that the fringe visibility seen at each homodyne between the local oscillator and squeezing path was above $98 \%$.

Each homodyne detector directly measures the variance of one of the two EPR squeezing modes. The detected noise is sent to an oscilloscope (Tektronix MDO3024) where the time series are recorded. The power spectrum density and Wiener filter are calculated from these time-series data and digital filtering of the signal and witness channel is done post data collection in the Fourier domain. The Wiener filter is calculated by taking the cross-spectral density between the signal and witness channels and normalizing it to the spectral density of the signal channel, as described in Eq. (2).

In combining the two channels a filter is specified: either the calculated Wiener filter or a filter constant in magnitude and phase (e.g., \pm 1 ). Equation (1) gives us the method of their application, where we allow $g$ to be either the Wiener filter or this simple linear filter. In applying a linear filter, its magnitude is chosen to maximize the level of squeezing recovered. This is done as the mode clearer cavity is not actively stabilized, leading to a changing optimum linear magnitude between subsequent time-series data. We see this in Figs. 3 and 4 where their linear filters have magnitudes of 0.85 and 0.9 , respectively.

There are a number of reasons why the Wiener filter provides optimal noise subtraction. As Eq. (2) suggests, it directly calculates the cross-spectral density of the noise between the two channels. This calculation accounts for all decoherence and phase shifts between the correlated noise, providing a numeric transfer function between the two channels. In the results presented here, we can see how this filter provides optimal performance. First, in the case of the frequencydependent squeezing spectra, the Wiener filter demonstrates its tracking of the rotating squeezing ellipse. Second, in the case of our lossy system, the Wiener filter accounts for the decoherence between the two channels. This ensures that the digital processing of the two channels does not add additional noise to our readout, providing the optimal noise cancellation. In our frequency-dependent results we show the applied Wiener filter together with a model of the optimum filter for the corresponding configuration. 
(a)

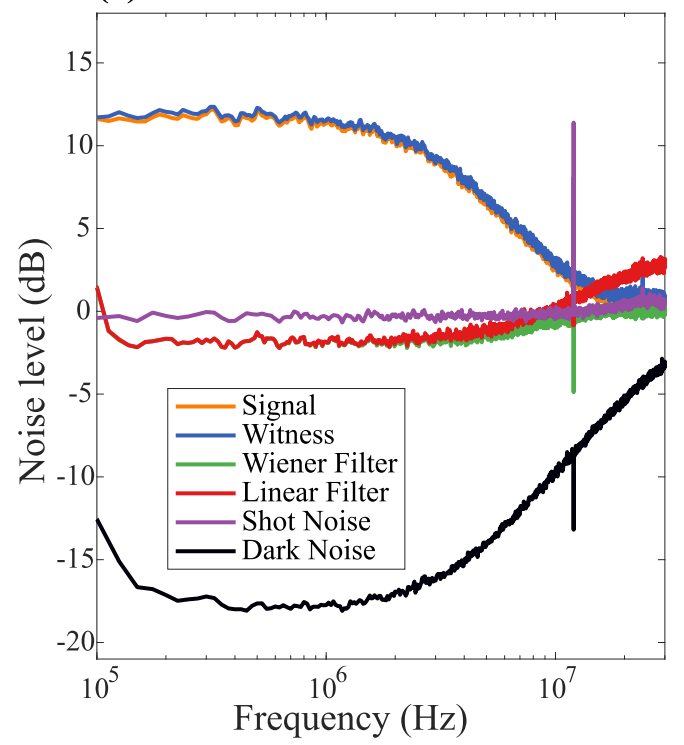

(b)
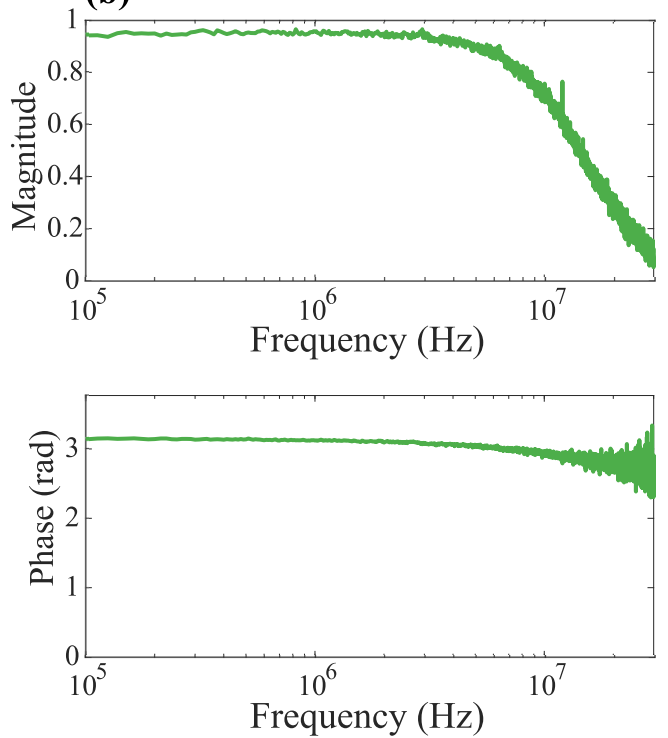

FIG. 2. (a) Noise spectra normalized to the shot noise of a single homodyne detector. The test cavity in this case is far detuned for both signal and witness fields. The purple trace shows the shot noise seen by the other homodyne. The black trace shows the dark noise of the measurement system. The raw signal (orange) and witness (blue) channels show the variance of each entangled field. These fields are are both approximately $12 \mathrm{~dB}$ above the shot-noise level, their slight difference due to the difference in losses on the two squeezing paths caused by the mode cleaner tuning. The two sub-shot-noise traces show a linear filter of -0.95 (red) and the Wiener filter (green). (b) Magnitude and phase of the Wiener filter.

\section{RESULTS AND DISCUSSION}

Figure 2, showing several spectra normalized to shot noise and the applied Wiener filter, displays the case without any rotation of the squeezing ellipse. The test cavity is far detuned from both the signal and witness fields so that the dispersion effects of the test cavity are not seen by the fields and there is no frequency-dependent rotation in the bandwidth of interest. As expected for a case without frequency dependence, the linear filter and Wiener filter performances are largely the same. The raw signal and witness channels have a variance $12 \mathrm{~dB}$ greater than that of shot noise and they show very similar noise features owing to their simultaneous creation in the OPO and similar beam path up until their separation at the (a)

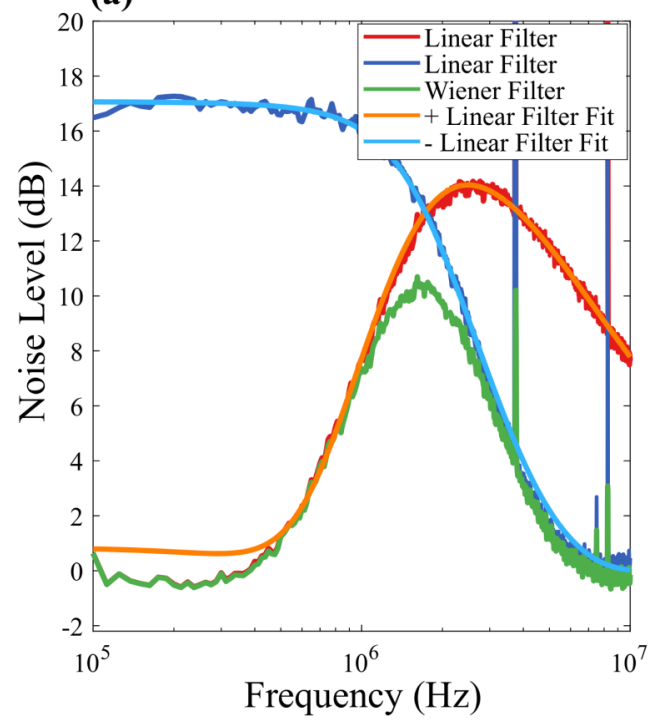

(b)
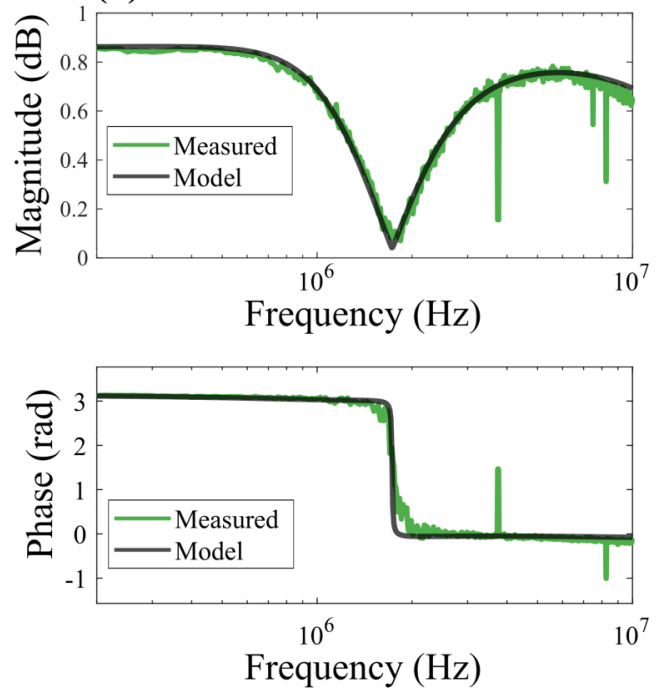

FIG. 3. (a) Noise spectra normalized to the shot noise of a single homodyne detector. Both signal and witness fields are detuned by half the linewidth of the test cavity. The signal and witness fields undergo the same phase change resulting in a rotation of the squeezed quadrature around the test cavity linewidth. The two linear filters are shown together with the results of a fitting routine [43]. In dark blue and light blue we have the -0.85 filter spectrum and its fitted model; in red and orange we have the +0.85 filter spectrum and its model. (b) Applied Wiener filter magnitude and phase shown together with a model that uses the parameters produced by the fitting routine. 
(a)

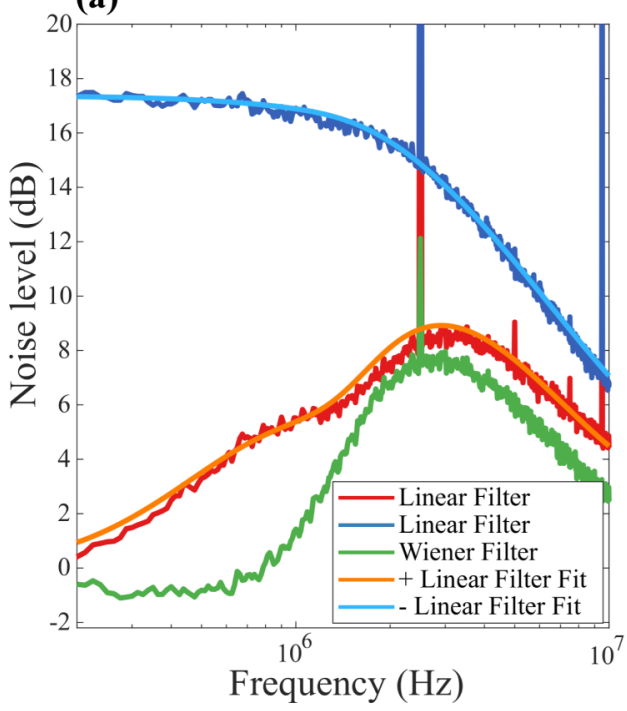

(b)
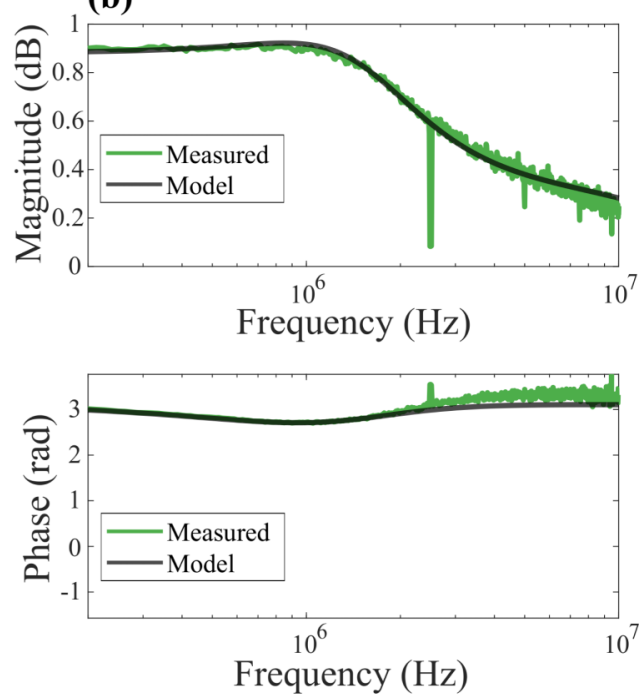

FIG. 4. (a) Noise spectra normalized to the shot noise of a single homodyne detector. The test cavity has here been tuned such that the signal field is resonant while the witness is detuned. The two linear filters are shown together with the results of a fitting routine [43]. In dark blue and light blue we have the -0.9 filter spectrum and its fitted model; in red and orange we have the +0.9 filter spectrum and its model. (b) Magnitude and phase of the Wiener filter shown together with its model taking the parameters from the fitting routine.

mode cleaner. These two channels, combined via digital signal processing in a linear fashion, result in a sub-shot-noise trace. This linear combination of the signal and witness is able to cancel quantum noise at frequencies below the linewidth of the OPO where the coherence between the signal and witness channel is high. Above the linewidth of the OPO, there is no cancellation. At these frequencies the coherence between the two channels falls and the linear filter begins to increase the noise on the channel. The direct subtraction or addition of two uncorrelated noise sources results in noise $3 \mathrm{~dB}$ higher than that of a single channel. Around the OPO linewidth the Wiener filter accounts for the appropriate coherence between the two channels, converging to the shot noise measured by a single photodiode. In this case the signal and witness fields, far detuned from the test cavity and thus unaffected by its intracavity loss, see the propagation and detection loss totaling at $33 \pm 2 \%$. Both linear and Wiener filters are able to recover the $2 \mathrm{~dB}$ of squeezing within our measurement band. The level of squeezing seen here demonstrates directly the squeezing performance of the OPO under the detection and propagation loss as well as the $3-\mathrm{dB}$ loss intrinsic to a two-detector system. The recovery of $2 \mathrm{~dB}$ of squeezing is not a fundamental limit to the digital signal processing technique but rather a limit set by the loss in our system. Figure 3 shows a case where both signal and witness fields are detuned by half the linewidth of the test cavity. In this case, the signal and witness fields undergo the same phase change resulting in a rotation of the squeezed quadrature around the test cavity linewidth. The roll-off in the positive linear filter seen here is due to the OPO linewidth. The Wiener filter is able to provide quantum noise cancellation, lying below the shot-noise level at lower frequencies and reducing to shot noise at higher frequencies above the linewidth of the OPO. Around the test cavity linewidth the signal and witness fields are each rotated by $45^{\circ}$; this rotation sees the homodynes detecting quadratures that are separated by $90^{\circ}$. Here the witness homodyne is detecting a quadrature that is completely uncorrelated with the quadrature detected by the signal homodyne. At this point the Wiener filter has a notch in its magnitude, ensuring that no additional noise is added. The notch and phase flip closely follow the expected Wiener filter, modeled using the fitted parameters. Through the fitting routine [43] used here we estimate the losses in this case to be $38 \pm 2 \%$, an increase from that seen in Fig. 2 due to the intracavity loss of the test cavity which is in agreement with a direct measurement of loss.

Figure 4 shows a case where the signal field is resonant and the witness field is detuned by a linewidth of the test cavity. The performance of the Wiener filter over a linear filter is highlighted between 0.5 and $1 \mathrm{MHz}$, where the Wiener filter appropriately accounts for the optimum phase and we see the recovery of sub-shot-noise sensitivity. Similar to the effect seen in Fig. 3, the rotation of the witness field sees the detection of correlated quadratures at low frequency and uncorrelated quadratures at high frequency. As we pass from correlated to uncorrelated there is no large phase change or inversion in the Wiener filter but rather a small phase tracking and a drop in magnitude so as not to add additional noise into the readout spectrum. These aspects of the Wiener filter are shown in both the model and applied Wiener filter. This configuration is of particular interest for its application to gravitational wave detectors. In a gravitational wave detector the additional effects of radiation pressure noise, which are absent in this tabletop demonstration, would cause the frequency-dependent rotation of the squeezed state back to a case where the readout of the detector would have consistent improvement across its sensitivity band.

The rotation in the squeezed state generated by having only the witness field detuned by a linewidth of the test cavity is exactly the rotation proposed in [40]. This rotation, together with the application of the Wiener filtering technique, completes the proof-of-principle experiment of applying EPR entanglement to a gravitational wave detector. The tabletop 
experiment we present here reports low levels of squeezing due to large optical losses and low-efficiency detection. Applied in a gravitational wave detector with typical losses and squeezing levels, the EPR squeezing method together with Wiener filtering would reduce the quantum noise across the entire detector readout frequency range, without the need for additional long-baseline low-loss cavities.

\section{CONCLUSION}

In this paper we demonstrated that through Wiener filtering the independent detection of two EPR modes, we can achieve an optimal frequency-dependent squeezing profile. Without the intracavity loss introduced by the test cavity, we achieved an overall cancellation of quantum noise to a level $2 \mathrm{~dB}$ below the shot noise of a single detector, limited by optical and detection loss. When test cavity dynamics were introduced we highlighted the robustness of Wiener filtering in the presence of this dispersive and lossy system. This filtering technique is particularly relevant for informing quantum noise reduction strategies for future gravitational wave detectors and has application in other quantum metrology experiments.

The data that support the plots within this paper and other findings of this study are available from the corresponding author upon reasonable request.

\section{ACKNOWLEDGMENTS}

This research was supported by the Australian Research Council under the ARC Centre of Excellence for Gravitational Wave Discovery, Grant No. CE170100004. D.W.G. would like to acknowledge funding from the Australian Government Research Training Program Scholarship for his research. The authors would like to thank Daniel Töyrä, Paul Altin, and Sheon Chua for valuable discussions and Mikhail Korobko for valuable comments in the review process.

The authors declare no competing interests.
[1] N. Wiener, Extrapolation, Interpolation, and Smoothing of Stationary Time Series (MIT Press, Cambridge, 1964).

[2] S. V. Vaseghi, Advanced Signal Processing and Digital Noise Reduction (Vieweg+Teubner, Wiesbaden, 1996), pp. 140-163.

[3] C. Meng, G. A. Brawley, J. S. Bennett, M. R. Vanner, and W. P. Bowen, Mechanical Squeezing via Fast Continuous Measurement, Phys. Rev. Lett. 125, 043604 (2020).

[4] N. Matsumoto and N. Yamamoto, Preparing mechanical squeezing of a macroscopic pendulum near quantum regimes, arXiv:2008.10848.

[5] H. Müller-Ebhardt, H. Rehbein, C. Li, Y. Mino, K. Somiya, R. Schnabel, K. Danzmann, and Y. Chen, Quantum-state preparation and macroscopic entanglement in gravitational-wave detectors, Phys. Rev. A 80, 043802 (2009).

[6] H. Miao, S. Danilishin, H. Müller-Ebhardt, H. Rehbein, K. Somiya, and Y. Chen, Probing macroscopic quantum states with a sub-Heisenberg accuracy, Phys. Rev. A 81, 012114 (2010).

[7] R. DeRosa, J. C. Driggers, D. Atkinson, H. Miao, V. Frolov, M. Landry, J. A. Giaime, and R. X. Adhikari, Global feed-forward vibration isolation in a $\mathrm{km}$ scale interferometer, Class. Quantum Grav. 29, 215008 (2012)

[8] M. Coughlin, J. Harms, N. Christensen, V. Dergachev, R. DeSalvo, S. Kandhasamy, and V. Mandic, Wiener filtering with a seismic underground array at the Sanford Underground Research Facility, Class. Quantum Grav. 31, 215003 (2014).

[9] F. Badaracco, J. Harms, A. Bertolini, T. Bulik, I. Fiori, B. Idzkowski, A. Kutynia, K. Nikliborc, F. Paoletti, A. Paoli, L. Rei, and M. Suchinski, Machine learning for gravitationalwave detection: Surrogate Wiener filtering for the prediction and optimized cancellation of Newtonian noise at Virgo, Class. Quantum Grav. 37, 195016 (2020).

[10] D. Davis, T. Massinger, A. Lundgren, J. C. Driggers, A. L. Urban, and L. Nuttall, Improving the sensitivity of advanced LIGO using noise subtraction, Class. Quantum Grav. 36, 055011 (2019).

[11] The LIGO Scientific Collaboration, J. Aasi, B. P. Abbott, R. Abbott, T. Abbott, M. R. Abernathy, K. Ackley, C. Adams,
T. Adams, P. Addesso, R. X. Adhikari et al., Advanced LIGO, Class. Quantum Grav. 32, 074001 (2015).

[12] F. Acernese, M. Agathos, K. Agatsuma, D. Aisa, N. Allemandou, A. Allocca, J. Amarni, P. Astone, G. Balestri, G. Ballardin et al., Advanced Virgo: A second-generation interferometric gravitational wave detector, Class. Quantum Grav. 32, 024001 (2014).

[13] T. Akutsu, M. Ando, K. Arai, Y. Arai, S. Araki, A. Araya, N. Aritomi, Y. Aso, S. W. Bae, Y. B. Bae et al., Overview of KAGRA: Detector design and construction history, Prog. Theor. Exp. Phys. 2021, 05A101 (2021).

[14] H. Lück, C. Affeldt, J. Degallaix, A. Freise, H. Grote, M. Hewitson, S. Hild, J. Leong, M. Prijatelj, K. A. Strain, B. Willke, H. Wittel, and K. Danzmann, The upgrade of GEO 600, J. Phys.: Conf. Ser. 228, 012012 (2010).

[15] B. P. Abbott, R. Abbott, T. D. Abbott, F. Acernese, K. Ackley, C. Adams, T. Adams, P. Addesso, R. X. Adhikari, V. B. Adya et al., GW170817: Observation of Gravitational Waves from a Binary Neutron Star Inspiral, Phys. Rev. Lett. 119, 161101 (2017).

[16] B. P. Abbott, R. Abbott, T. D. Abbott, F. Acernese, K. Ackley, C. Adams, T. Adams, P. Addesso, R. X. Adhikari, V. B. Adya et al., Multi-messenger observations of a binary neutron star merger, Astrophys. J. 848, L12 (2017).

[17] V. B. Adya, M. J. Yap, D. Töyrä, T. G. McRae, P. A. Altin, L. K. Sarre, M. Meijerink, N. Kijbunchoo, B. J. J. Slagmolen, R. L. Ward, and D. E. McClelland, Quantum enhanced kHz gravitational wave detector with internal squeezing, Class. Quantum Grav. 37, 07LT02 (2020).

[18] K. Ackley, V. B. Adya, P. Agrawal, P. Altin, G. Ashton, M. Bailes, E. Baltinas, A. Barbuio, D. Beniwal, C. Blair et al., Neutron star extreme matter observatory: A kilohertz-band gravitational-wave detector in the global network, Publ. Astron. Soc. Aust. 37, e047 (2020).

[19] M. Evans, R. X. Adhikari, C. Afle, S. W. Ballmer, S. Biscoveanu, S. Borhanian, D. A. Brown, Y. Chen et al., A horizon study for cosmic explorer science, observatories, and community, arXiv:2109.09882v2. 
[20] J. van den Brand, Proceedings of the 11th Einstein Symposium, 2020 (unpublished), available at https://indico.in2p3.fr/ event/20576/contributions/88527/attachments/61174/83351/ Einstein_Telescope_Site_Infrastructure.pdf.

[21] C. M. Caves, Quantum-mechanical noise in an interferometer, Phys. Rev. D 23, 1693 (1981).

[22] A. Buikema, C. Cahillane, G. L. Mansell, C. D. Blair, R. Abbott, C. Adams, R. X. Adhikari, A. Ananyeva, S. Appert, K. Arai et al., Sensitivity and performance of the Advanced LIGO detectors in the third observing run, Phys. Rev. D 102, 062003 (2020).

[23] H. J. Kimble, Y. Levin, A. B. Matsko, K. S. Thorne, and S. P. Vyatchanin, Conversion of conventional gravitational-wave interferometers into quantum nondemolition interferometers by modifying their input and/or output optics, Phys. Rev. D 65, 022002 (2001).

[24] C. M. Caves, Quantum-Mechanical Radiation-Pressure Fluctuations in an Interferometer, Phys. Rev. Lett. 45, 75 (1980).

[25] V. B. Braginskii, Classical and quantum restrictions on the detection of weak disturbances of a macroscopic oscillator, $\mathrm{Zh}$. Eksp. Teor. Fiz. 53, 1434 (1967) [Sov. Phys. JETP 26, 831 (1968)]

[26] F. Acernese, M. Agathos, L. Aiello, A. Ain, A. Allocca, A. Amato, S. Ansoldi, S. Antier, M. Arène, N. Arnaud et al., Quantum Backaction on kg-Scale Mirrors: Observation of Radiation Pressure Noise in the Advanced Virgo Detector, Phys. Rev. Lett. 125, 131101 (2020).

[27] M. J. Yap, J. Cripe, G. L. Mansell, T. G. McRae, R. L. Ward, B. J. J. Slagmolen, P. Heu, D. Follman, G. D. Cole, T. Corbitt, and D. E. McClelland, Broadband reduction of quantum radiation pressure noise via squeezed light injection, Nat. Photon. 14, 19 (2020).

[28] H. Yu, L. McCuller, M. Tse, N. Kijbunchoo, L. Barsotti, N. Mavalvala, J. Betzwieser, C. D. Blair, S. E. Dwyer, A. Effler et al., Quantum correlations between light and the kilogrammass mirrors of LIGO, Nature (London) 583, 43 (2020).

[29] A. Buonanno and Y. Chen, Quantum noise in second generation, signal-recycled laser interferometric gravitational-wave detectors, Phys. Rev. D 64, 042006 (2001).

[30] H. Grote, K. Danzmann, K. L. Dooley, R. Schnabel, J. Slutsky, and H. Vahlbruch, First Long-Term Application of Squeezed States of Light in a Gravitational-Wave Observatory, Phys. Rev. Lett. 110, 181101 (2013).

[31] J. Aasi, J. Abadie, B. P. Abbott, R. Abbott, T. D. Abbott, M. R. Abernathy, C. Adams, T. Adams, P. Addesso, R. X. Adhikari et al., Enhanced sensitivity of the LIGO gravitational wave detector by using squeezed states of light, Nat. Photon. 7, 613 (2013).

[32] F. Acernese, M. Agathos, L. Aiello, A. Allocca, A. Amato, S. Ansoldi, S. Antier, M. Arène, N. Arnaud, S. Ascenzi et al., Increasing the Astrophysical Reach of the Advanced Virgo Detector via the Application of Squeezed Vacuum States of Light, Phys. Rev. Lett. 123, 231108 (2019).
[33] M. Tse, H. Yu, N. Kijbunchoo, A. Fernandez-Galiana, P. Dupej, L. Barsotti, C. D. Blair, D. D. Brown, S. E. Dwyer et al., Quantum-Enhanced Advanced LIGO Detectors in the Era of Gravitational-Wave Astronomy, Phys. Rev. Lett. 123, 231107 (2019).

[34] E. Oelker, T. Isogai, J. Miller, M. Tse, L. Barsotti, N. Mavalvala, and M. Evans, Audio-Band Frequency-Dependent Squeezing for Gravitational-Wave Detectors, Phys. Rev. Lett. 116, 041102 (2016).

[35] E. Capocasa, M. Barsuglia, J. Degallaix, L. Pinard, N. Straniero, R. Schnabel, K. Somiya, Y. Aso, D. Tatsumi, and R. Flaminio, Estimation of losses in a $300 \mathrm{~m}$ filter cavity and quantum noise reduction in the KAGRA gravitational-wave detector, Phys. Rev. D 93, 082004 (2016).

[36] Y. Zhao, N. Aritomi, E. Capocasa, M. Leonardi, M. Eisenmann, Y. Guo, E. Polini, A. Tomura, K. Arai, Y. Aso, Y.-C. Huang, R.-K. Lee, H. Lück, O. Miyakawa, P. Prat, A. Shoda, M. Tacca, R. Takahashi, H. Vahlbruch, M. Vardaro, C.-M. Wu, M. Barsuglia, and R. Flaminio, Frequency-Dependent Squeezed Vacuum Source for Broadband Quantum Noise Reduction in Advanced Gravitational-Wave Detectors, Phys. Rev. Lett. 124, 171101 (2020).

[37] P. Kwee, J. Miller, T. Isogai, L. Barsotti, and M. Evans, Decoherence and degradation of squeezed states in quantum filter cavities, Phys. Rev. D 90, 062006 (2014).

[38] N. Aritomi, M. Leonardi, E. Capocasa, Y. Zhao, and R. Flaminio, Control of a filter cavity with coherent control sidebands, Phys. Rev. D 102, 042003 (2020).

[39] E. Capocasa, Y. Guo, M. Eisenmann, Y. Zhao, A. Tomura, K. Arai, Y. Aso, M. Marchiò, L. Pinard, P. Prat, K. Somiya, R. Schnabel, M. Tacca, R. Takahashi, D. Tatsumi, M. Leonardi, M. Barsuglia, and R. Flaminio, Measurement of optical losses in a high-finesse $300 \mathrm{~m}$ filter cavity for broadband quantum noise reduction in gravitational-wave detectors, Phys. Rev. D 98, 022010 (2018)

[40] Y. Ma, H. Miao, B. H. Pang, M. Evans, C. Zhao, J. Harms, R. Schnabel, and Y. Chen, Proposal for gravitational-wave detection beyond the standard quantum limit through EPR entanglement, Nat. Phys. 13, 776 (2017).

[41] M. J. Yap, P. Altin, T. G. McRae, B. J. J. Slagmolen, R. L. Ward, and D. E. McClelland, Generation and control of frequency-dependent squeezing via EinsteinPodolsky-Rosen entanglement, Nat. Photon. 14, 223 (2020).

[42] J. Südbeck, S. Steinlechner, M. Korobko, and R. Schnabel, Demonstration of interferometer enhancement through Einstein-Podolsky-Rosen entanglement, Nat. Photon. 14, 240 (2020).

[43] C. Avinadav, Multiple curve fitting with common parameters using NLINFIT, https://www.mathworks.com/matlabcentral/ fileexchange/40613-multiple-curve-fitting-with-commonparameters-using-nlinfi, version 1.1.0.0 (MathWorks, Natick, 2016). 